\title{
IVISEM, una Medida de la Violencia Social Encubierta hacia las Mujeres
}

\author{
IVISEM, a Measure of Covert Social Violence against Women
}

\author{
Agustina M. Vinagre-González ${ }^{1}$, Marta E. Aparicio-García y Jesús M. Alvarado-Izquierdo³
}

\begin{abstract}
Resumen
El objetivo de esta investigación ha sido diseñar y validar el Inventario de Violencia Social Encubierta hacia las mujeres (IVISEM). El inventario evalúa siete dimensiones relacionadas con los mandatos de género que la mujer asume como algo normalizado y que de algún modo la someten a la figura masculina, produciéndose una forma de victimización, aceptada socialmente, que hemos denominado Violencia Social Encubierta hacia las mujeres. La construcción y validación del inventario se realizó en tres etapas: (1) elaboración y revisión de 155 ítems; (2) una primera versión con 81 ítems aplicada a una muestra de 2.168 personas y (3) una versión definitiva de 35 ítems administrada a 4.400 mujeres. El inventario mostró una adecuada fiabilidad y se comprobó el ajuste de su estructura a través de un Análisis Factorial Confirmatorio. La relación con otras variables que miden constructos similares permite concluir que el IVISEM es un instrumento adecuado para la detección de distintas formas de discriminación y violencia hacia las mujeres.
\end{abstract}

Palabras clave: socialización diferencial, violencia social encubierta, mujeres, mandatos de género, instrumento de evaluación

\begin{abstract}
The main aim of this research has been to design and validate the Inventory of Covert Social Violence against Women (IVISEM). The inventory evaluates seven dimensions related to gender roles that woman assumes as something normalized and that somehow subject her to the masculine figure, producing a form of victimization, socially accepted, that we have called Covert Social Violence against them. The construction and validation of the inventory was carried out in three stages: (1) elaboration and review of 155 items by experts; (2) a first version with 81 items applied to a sample of 2,168 people and (3) a final version of 35 items administered to 4,400 women. The inventory showed adequate reliability and the adjustment of its structure was checked through a Confirmatory Factor Analysis. The relationship with other variables that measure similar constructs allows us to conclude that the IVISEM is an adequate instrument for the detection of different forms of discrimination and violence against women.
\end{abstract}

Keywords: differential socialization, social violence, women, gender mandates, evaluation instrument

\footnotetext{
${ }^{1}$ Dra en Estudios de Género. Profesora de la Facultad de Derecho. Grado de Criminología. Universidad Internacional de La Rioja (UNIR), España.

${ }^{2}$ Dra en Psicología. Profesora Titular. Facultad de Psicología. Universidad Complutense de Madrid. Facultad de Psicología. Universidad Complutense de Madrid. Campus de Somosaguas, s/n. 28223. Madrid, España. Correo: aparicio@psi.ucm.es (Correspondencia)

${ }^{3}$ Dr en Psicología. Profesor titular en la Facultad de Psicología de la Universidad Complutense de Madrid, España.

Revista Iberoamericana de Diagnóstico y Evaluación - e Avaliação Psicológica. RIDEP · N57 · Vol.4 · 149-161 · 2020

ISSN: 1135-3848 print /2183-6051online
} 


\section{Introducción}

La violencia de género que sufren las mujeres va más allá de la violencia explícita y física que se contabiliza en las estadísticas (Médicos del Mundo, 2018; Perez-Viejo et al., 2011). La mayor parte de la investigación científica se ha centrado en analizar la violencia física y psicológica hacia las mujeres en diferentes ámbitos (López-Cepero, Rodríguez-Franco, \& Rodríguez-Díaz, 2015; Johnson, 2006; Muñoz \& Echeburúa, 2016; Sackett \& Sounders, 2001). Sin embargo, existen estudios que remarcan la importancia de otras formas de violencia encubiertas que pueden conllevar consecuencias negativas importantes para quien las padece (Bonino, 2008). Se trata de formas de violencia sociales y estructurales (Bonino, 1996, 2008; Bourdieu, 2000; Galtung, 1990, 2016). En este sentido, las mujeres están sometidas a un tipo de violencia social que tiene que ver con los mandatos de género que la sociedad promueve (Casado-Mejía \& GarcíaCarpintero, 2018). Dichos mandatos de género hacen referencia a las normas sociales que marcan qué papel deben desempeñar los hombres y las mujeres en la sociedad para ser poder ser considerados como mujeres-femeninas $u$ hombres-masculinos (Hernando, 2007; Lagarde, 1990, 2003). Estos mandatos se interiorizan en edades tempranas en hombres y mujeres a través de la socialización diferencial (Etchezahar, 2014; Ferrer-Pérez \& Bosch-Fiol, 2014, 2019), y suponen la asimilación de pautas sociales de género establecidas que dan lugar a la aceptación y asunción de los mandatos de género como algo normalizado (Colás, 2007), siendo estas normas o mandatos de género más restrictivas para las niñas que para los niños (Blum, Mmari, \& Moreau, 2017). Diferentes autores/as consideran, incluso, que la feminidad tradicional hegemónica se constituye como factor de riesgo para la salud (Bonino, 2008; Lagarde, 2006). Según el modelo explicativo de la violencia de género de BoschFiol y Ferrer-Pérez (2013), la socialización diferencial va generando diferencias $y$ desigualdades entre hombres y mujeres (BoschFiol et al., 2013; Pérez-Viejo et. al, 2011) que se ven reflejados en los procesos de violencia ejercidos contra las mujeres (Morillas, Patró, \& Aguilar, 2014) y que son difíciles de reconocer a no ser que esta violencia sea explícita y física (Delegación del Gobierno para la Violencia de Género, 2015). Otros estudios han analizado algunos de los mandatos de género y su relación con la salud en la mujer (Anastasiadou, Aparicio, Sepúlveda, \& Sánchez-Beleña, 2013; AparicioGarcía \& Alvarado-Izquierdo, 2018).

Estas consecuencias negativas en la mujer se pueden llegar a considerar como una forma de violencia "encubierta" ya que conlleva cierta forma de victimización en las mujeres. Por ejemplo, algunos estudios subrayan que la violencia sutil de género no es considerada en la misma medida que la violencia manifiesta como constitutiva de agresión y, por tanto, no se detecta como violencia, aun siéndolo (Novo, Herbón, \& Amado, 2016). Aunque existen discrepancias en cuanto a la definición del término violencia, recientes teorías señalan que existen situaciones en las que, aunque no se busque intencionalmente el daño de otra persona, sí se puede provocar dicho daño a medio y largo plazo como consecuencia del mantenimiento de ciertos privilegios o desigualdades (Martínez-Pacheco, 2016). Algunas de estas formas de violencia serían la violencia moral de Segato (2003) o la violencia simbólica de Bourdieu (2000) (JiménezBautista, 2012; Martínez-Pacheco, 2016).

De hecho, el modelo piramidal de Bosch-Fiol y Ferrer-Pérez (2013) tiene en cuenta la socialización como parte de la explicación de las formas de violencia hacia las mujeres, tanto de las formas de violencia explícitas como de aquellas más sutiles o encubiertas.

En este marco presentamos el constructo "Violencia Social Encubierta hacia las Mujeres", que se entiende como consecuencia de asumir determinados mandatos de género en base al proceso de socialización diferencial. Esto responde a la demanda de diferentes autoras que afirman que falta investigación académica en relación con formas de violencia encubiertas para poder trabajar en la prevención de todas las formas de violencia hacia las mujeres (Ellsberg et al., 2015; Temmerman, 2015). En el marco de las formas de violencia sutiles o encubiertas (Bourdieu, 2000; Galtung, 2016), en las que el aspecto fundamental no es la intención sino las consecuencias que la situación supone para quien la sufre, se define la Violencia Social Encubierta 
hacia las Mujeres como la victimización que están sufriendo las mujeres en base a la socialización diferencial que les lleva a asumir como algo normalizado el papel que les asigna la sociedad en relación con tareas de cuidado, deberes maternales, estéticos, etc. (Vinagre, 2019). Este rol, basado en los estereotipos de género y la socialización diferencial, puede llegar a tener consecuencias negativas en las mujeres (CasadoMejía \& García-Carpintero, 2018; Ferrer-Pérez \& Bosch-Fiol, 2014; Latorre, López, Izquierdo, \& García, 2018; Novo et al., 2016; Perez-Viejo et al., 2011).

El objetivo la investigación presentada es el diseño y validación de un instrumento que evalúe la violencia social encubierta hacia las mujeres en base a la socialización diferencial interiorizada o asumida. Aunque existe un número importante de escalas y cuestionarios que miden distintas formas de violencia hacia las mujeres, la mayor parte de ellos se centra en la violencia explícita hacia las mujeres en la relación de pareja. Sin embargo, son varias las investigaciones que concluyen la necesidad de trabajar en nuevos instrumentos que ayuden tanto a las mujeres como a los profesionales a tomar conciencia de dicha situación de violencia encubierta (García-Moreno et al., 2015ab; Novo et al., 2016; RodríguezBlanes, Vives-Cases, Miralles-Bueno, San Sebastián, \& Goicolea, 2017).

Existen diferentes instrumentos psicométricos que se centran en la percepción social que se tiene en relación con el sexo biológico y los roles asignados a uno u otro sexo según el estereotipo asumido. Por ejemplo, la Escala de Actitudes Sexistas hacia la Mujer SATWS (Benson \& Vicent, 1980), tiene como objetivo evaluar las actitudes que sitúan a las mujeres en situación de inferioridad con respecto a los hombres. Las autoras se centran en 6 dimensiones que indicarían sexismo, pero no lo relacionan con una forma de violencia o victimización. La Escala de Creencias Patriarcales PBS (Smith, 1990) se centra en comprobar la posible relación entre la presencia de una ideología patriarcal y una mayor probabilidad de ejercer maltrato contra las mujeres en la relación de pareja. Tiene en cuenta únicamente la interiorización de la legitimización de la autoridad masculina y la consecuente sumisión de las mujeres. El Inventario de Sexismo
Ambivalente ASI (Expósito, Moya, \& Glick, 1998; Glick \& Fiske 1996) mide dos formas de sexismo: el hostil y el benevolente, siendo esta forma de sexismo entendida como aceptación de la dependencia y debilidad de las mujeres con respecto a los hombres. Por último, el Inventario de Conformidad con las Normas de Género Femeninas CFNI (Mahalik et al., 2005), cuenta con varias dimensiones que, aunque tienen en cuenta la conformidad con el rol femenino por parte de las mujeres, no llega a profundizar en la interiorización de este ni lo entiende como una forma de violencia encubierta.

Estos instrumentos recogen, cada uno de ellos, algunos aspectos relacionados con actitudes sexistas en base a roles y estereotipos de género, pero no tienen en cuenta en conjunto los mandatos de género que se presentan en este estudio y que la mayoría de investigaciones relacionan con una mayor probabilidad de generar consecuencias negativas en las mujeres (Bareket, Kahalon, Shnabel, \& Glick, 2018; Ferrer-Pérez \& BoschFiol, 2019; Perez-Viejo et al., 2011; Simón, 2008; García-Calvente, Mateo-Rodríguez, \& MarotoNavarro, 2004; Lagarde, 2003) y, por tanto, pueden entenderse, en base a dichas consecuencias negativas, como una forma de violencia social encubierta o no explícita. (Bourdieu, 2000; Segato, 2003; Galtung, 2016).

El instrumento psicométrico que presentamos, a diferencia de los ya existentes, tiene en cuenta siete dimensiones planteadas en base a los mandatos de género que parecen estar más relacionados con la probabilidad de generar consecuencias negativas en las mujeres. Estos son: a) Mandatos de género relacionados con la maternidad (Casado-Mejía \& García-Carpintero, 2018; Lagarde, 2003); b) La idea de amor romántico y relación de pareja estable; (Bareket et al., 2018; Ferrer-Pérez \& Bosch-Fiol, 2014, 2019); c) El papel de mujer cuidadora (GarcíaCalvente et al., 2004; Perez-Viejo et al., 2011); d) La renuncia al desarrollo profesional (Conway, Bourque \& Scott, 2013; Simón, 2008; Varela, 2013); e) Las actitudes relacionadas con la sumisión (Bourdieu, 2000; Martínez, Bonilla, Gómez, \& Bayot, 2008); f) Las ideas preconcebidas en cuanto a aspectos biológicos y aptitudes básicamente femeninas (Carvajal-Lohr, Flores-Ramos, Marin, \& Morales, 2016; Vázquez, 
2013); y g) La auto convicción de la bondad de mantener las diferencias entre hombres y mujeres como algo "natural", también entendida como neosexismo (Delgado, Palma, \& Rivas, 2015; Moya, Expósito, \& Padilla, 2006).

Ninguno de los instrumentos existentes indicados anteriormente tiene en cuenta las siete dimensiones en conjunto, que mide el IVISEM. Los que miden aspectos más parecidos serían el Inventario de Sexismo Ambivalente (ASI), y el Inventario de Conformidad con las Normas de Género Femeninas (CFNI), pero no tienen en cuenta todas las dimensiones en un mismo instrumento y no lo relacionan con una forma de violencia social encubierta.

Detectamos, por tanto, la necesidad de validar un instrumento que pueda medir hasta qué punto las mujeres puede estar sufriendo esta denominada violencia social encubierta basada en la asunción de los mandatos de género anteriormente indicados y que formarían las siete dimensiones que mide el instrumento psicométrico.

\section{Método}

\section{Procedimiento y Participantes}

La construcción y validación del IVISEM se realizó en tres etapas:

Primera etapa (revisión de los ítems). La primera versión del IVISEM constó de 155 ítems. Estos ítems se redactaron a partir de nuestra experiencia clínica en intervención con mujeres que presentaban alguna alteración emocional relacionada con la socialización diferencial. La revisión teórica y gramatical de la escala contó con la evaluación de contenido por parte de un panel de 10 expertas en violencia de género, aspecto fundamental para la elaboración de un instrumento psicométrico (Grant \& Davis, 1997; Guarderas et al., 2018), usando el Método de Agregados Individuales (Corral, 2009). En esta fase se contó únicamente con mujeres puesto que son áreas donde mayoritariamente trabajan mujeres y el cuestionario va dirigido a esta población. Para aumentar la validez de contenido se planteó una segunda revisión por parte de 50 personas formadas en género que debían realizar una asignación ciega de ítems a cada dimensión. Después de este análisis se retuvieron los ítems con un acuerdo igual o mayor al $70 \%$ inter-jueces, reduciéndose el instrumento a 81 ítems.

Segunda etapa (primera investigación con la versión inicial). El cuestionario formado por 81 ítems, con respuestas en una escala Likert de $1=$ totalmente en desacuerdo a $5=$ totalmente de acuerdo, se aplicó a una muestra a través de la herramienta de formularios de la plataforma Google Forms. Para la difusión del enlace se utilizó la divulgación de este a través de las redes sociales, contactando con personas que tenían un número elevado de seguidoras (especialmente en Twitter) y que difundieron ampliamente la investigación. Las participantes aceptaban antes de entrar en la aplicación del cuestionario un consentimiento informado en el que se les indicaba de forma explícita el carácter anónimo de la prueba. Contestaron a esta versión inicial junto a un cuestionario de datos demográficos un total de 2168 mujeres con una media de edad de 31.7 años $(D T=12.02)$, de entre 18 y 79 años. El $70.8 \%$ eran solteras, el $22 \%$ casadas, el $5.8 \%$ separadas o divorciadas, el $0.9 \%$ eran viudas y el $0.6 \%$ no se definían. El 43\% trabajaba fuera de casa, el 28.6 $\%$ eran estudiantes, el $12.6 \%$ estudiaba y trabajaba, el $8.5 \%$ estaba en paro, el $2.4 \%$ se dedicaba sólo a las tareas domésticas, el $1.6 \%$ eran jubiladas o pensionistas y se definían como otras o no contestaban el 3.3\%. El 51.2\% tenía estudios universitarios terminados, el $45.3 \%$ estudios medios o bachillerato, el $3.4 \%$ estudios primarios y el 0.2 no contestan.

Tercera etapa (versión definitiva). Con los 35 ítems que mejor funcionamiento habían mostrado en la etapa anterior se administró el IVISEM (Ver Anexo I) junto a otros dos instrumentos a una muestra de 4400 mujeres, siguiendo el mismo procedimiento que en la etapa anterior. Esta segunda aplicación fue contestada por 4400 mujeres con una media de edad de 35.8 años $(D T=12.21)$, de entre 14 y 83 años. El 34.07 $\%$ eran solteras con pareja estable, el $30.27 \%$ casadas, el $27.20 \%$ solteras sin pareja estable, el $7.50 \%$ eran separadas o divorciadas y el $0.82 \%$ viudas. El $52.25 \%$ trabajaba fuera de casa, $17.82 \%$ eran estudiantes, el $11.05 \%$ estudiaba y trabajaba, el $7.61 \%$ estaba en paro, el $4.05 \%$ se dedicaba a las tareas domésticas, el $2.45 \%$ eran jubiladas o pensionistas y se definían como otras el $3.86 \%$. El 58.12\% tenía estudios universitarios 
terminados, el $37.82 \%$ estudios medios o bachillerato, el $3.86 \%$ estudios primarios y el $0.09 \%$ sin estudios.

\section{Instrumentos}

En todos los estudios se aplicó un cuestionario demográfico para recoger información sobre el género, edad, nivel de estudios, situación laboral y estado civil.

En la segunda investigación en la que se evaluó la relación del IVISEM con otras medidas de constructos similares se utilizaron los siguientes instrumentos:

Inventario de Sexismo Ambivalente (ASI; Glick \& Fiske, 1996, en su versión española de Expósito et al., 1998). Consta de 22 ítems que se responden con una escala Likert. El instrumento mide dos dimensiones relacionadas con la actitud sexista: sexismo hostil (SH) y sexismo benevolente (SB). El alfa de Cronbach total para esta muestra fue de .90 .

Inventario de Conformidad con las Normas de Género Femeninas (CFNI-45-r; Aparicio-García \& Alvarado-Izquierdo, 2019). Mide 9 normas de género que definen el comportamiento de las mujeres (Mahalik et al., 2005): Invertir en apariencia; Cuidado de niños/as; Doméstica; Modestia; Dulce y Agradable; Relaciones; Romántica; Fidelidad sexual y Delgadez. Las respuestas se recogen en una escala Likert de $1=$ Totalmente en desacuerdo a $4=$ Totalmente de acuerdo. El coeficiente alfa de Cronbach total para esta muestra es de .78 .

\section{Análisis de datos}

Para analizar la fiabilidad por consistencia interna se estimó mediante el coeficiente de Cronbach y el procedimiento Omega Jerárquico de McDonnald y GLB (gran límite inferior de la fiabilidad). Para evaluar la validez de constructo en relación a la estructura teórica propuesta se utilizaron técnicas de análisis factorial confirmatorio (AFC), siguiendo como guía las recomendaciones sobre los puntos de corte de $\mathrm{Hu}$ y Bentler (1999), que señalan que deben ser mayores a $.95 / .90$ para Índice de ajuste comparativo (CFI) e Índice de ajuste de TuckerLewis (TLI), y menores a $.05 / .08$ para Error cuadrático medio de aproximación (RMSEA), y menores a $.06 / .08$ para Residuo cuadrático medio estandarizado (SRMR) para un ajuste bueno o aceptable, respectivamente. Para tomar la decisión sobre la eliminación de ítems en la versión definitiva del instrumento se tuvo en cuenta tanto la discriminación de los ítems como la relevancia y representatividad de contenido (Brown, 2014; Kline, 2016).

Para analizar la validez convergente con medidas de constructos relacionados se utilizaron correlaciones de Pearson, teniendo en cuenta las indicaciones sobre el tamaño del efecto hechas por Cohen (1988), interpretando los valores desde .10 hasta .30 como pequeños, de .30 hasta .50 como medianos y como grandes si son iguales o mayores de .50 .

Los análisis se realizaron en $\mathrm{R}$ (versión 3.4.2).

\section{Resultados}

\section{Análisis estructural y validez de constructo}

Como se disponía de un modelo teórico heptadimensional se aplicó un AFC para evaluar la bondad de ajuste del instrumento inicial formado por 81 ítems, siendo esta aceptable: $\chi 2(3138)=24694, \quad p<.001, \quad$ CFI $=.95, \quad$ TLI $=.95$, RMSEA $=.057 \quad(.056, .057)$ y $\mathrm{SRMR}=.067$. No obstante, para mejorar la calidad de la medida se decidió, a partir del estudio de los índices de modificación, eliminar aquellos ítems con pesos factoriales <.4 y aquellos que saturaban significativamente en más de un factor o mostraban correlaciones entre errores. Tras esta depuración se obtuvo el IVISEM definitivo de 35 ítems que fue aplicado a una segunda muestra. Los resultados con esta muestra indican que la fiabilidad calculada mediante el coeficiente Alpha de Cronbach fue de .93, con un Omega total de .95 y un Omega Jerárquico de .74, esto es, un $74 \%$ de la varianza de la puntuación total puede ser atribuida a un factor general y el resto hasta el $95 \%$ se explican por la naturaleza multidimensional de la medida.

En cuanto a la estructura factorial, la bondad de ajuste al modelo heptadimensional teórico se puede considerar excelente, con un $\chi^{2}(539)=6355$, $p<.001, \mathrm{CFI}=.97$, TLI $=.97$, RMSEA $=.050(.048$, $.051)$ y el $\mathrm{SRMR}=.053$. Los pesos estandarizados se muestran en la Tabla 1 y las correlaciones entre factores en la Tabla 2. 
Tabla 1. Pesos estandarizados en valor absoluto del IVISEM definitivo

\begin{tabular}{|c|c|c|c|c|}
\hline & Peso estandarizado & Error Estándar & Valor-z & $\mathrm{P}(>|\mathrm{z}|)$ \\
\hline \multicolumn{5}{|c|}{ Maternidad } \\
\hline I1 & .530 & & & \\
\hline I8 & .583 & .038 & 28.692 & .000 \\
\hline $\mathrm{I} 15$ & .699 & .043 & 30.450 & .000 \\
\hline $\mathrm{I} 22$ & .482 & .037 & 24.819 & .000 \\
\hline I29 & .584 & .044 & 25.042 & .000 \\
\hline \multicolumn{5}{|c|}{ Amor romántico y pareja } \\
\hline $\mathrm{I} 2$ & .618 & & & \\
\hline I9 & .395 & .033 & 19.101 & .000 \\
\hline I16 & .689 & .038 & 29.436 & .000 \\
\hline $\mathrm{I} 23$ & .266 & .032 & 13.625 & .000 \\
\hline $\mathrm{I} 30$ & .857 & .039 & 35.415 & .000 \\
\hline \multicolumn{5}{|c|}{ Cuidados } \\
\hline I3 & .634 & & & \\
\hline $\mathrm{I} 10$ & .760 & .024 & 49.930 & .000 \\
\hline I17 & .676 & .024 & 43.807 & .000 \\
\hline $\mathrm{I} 24$ & .696 & .025 & 44.399 & .000 \\
\hline I31 & .710 & .026 & 43.203 & .000 \\
\hline \multicolumn{5}{|c|}{ Proyección laboral } \\
\hline I4 & .742 & & & \\
\hline I11 & .630 & .023 & 37.102 & .000 \\
\hline $\mathrm{I} 18$ & .638 & .019 & 45.050 & .000 \\
\hline $\mathrm{I} 25$ & .370 & .020 & 24.871 & .000 \\
\hline $\mathrm{I} 32$ & .845 & .021 & 53.829 & .000 \\
\hline \multicolumn{5}{|c|}{ Actitudes y Sumisión } \\
\hline I5 & .461 & & & \\
\hline $\mathrm{I} 12$ & .553 & .050 & 24.117 & .000 \\
\hline I19 & .642 & .057 & 24.606 & .000 \\
\hline I26 & .598 & .058 & 22.531 & .000 \\
\hline I33 & .659 & .061 & 23.451 & .000 \\
\hline \multicolumn{5}{|c|}{ Biología y Aptitudes } \\
\hline I6 & .565 & & & \\
\hline $\mathrm{I} 13$ & .534 & .034 & 28.171 & .000 \\
\hline $\mathrm{I} 20$ & .677 & .038 & 31.923 & .000 \\
\hline $\mathrm{I} 27$ & .459 & .032 & 25.791 & .000 \\
\hline I34 & .738 & .040 & 32.739 & .000 \\
\hline \multicolumn{5}{|c|}{ Neosexismo } \\
\hline I7 & .651 & & & \\
\hline $\mathrm{I} 14$ & .716 & .032 & 34.344 & .000 \\
\hline I21 & .863 & .030 & 43.904 & .000 \\
\hline I 28 & .820 & .029 & 43.302 & .000 \\
\hline I35 & .911 & .032 & 43.495 & .000 \\
\hline
\end{tabular}

Tabla 2. Correlaciones entre las puntuaciones observadas de las escalas del IVISEM

\begin{tabular}{|c|c|c|c|c|c|c|c|c|}
\hline & Mat & Par & Cuid & Proy & Sum & Biol & Neo & IVISEM Total \\
\hline Mat & 1 & $.39 * *$ & $.48 * *$ & $.49 * *$ & $.31 * *$ & $.47 * *$ & $.32 * *$ & $.70 * *$ \\
\hline Par & & 1 & $.34 * *$ & $.38 * *$ & $.30 * *$ & $.38 * *$ & $.37 * *$ & $.62 * *$ \\
\hline Cuid & & & 1 & $.61 * *$ & $.57 * *$ & $.58 * *$ & $.30 * *$ & $.79 * *$ \\
\hline Proy & & & & 1 & $.51 * *$ & $.50 * *$ & $.35 * *$ & $.76^{* *}$ \\
\hline Sum & & & & & 1 & $.54 * *$ & $.23 * *$ & $.69 * *$ \\
\hline Biol & & & & & & 1 & $.38 * *$ & $.78 * *$ \\
\hline $\mathrm{Neo}$ & & & & & & & 1 & $.61 * *$ \\
\hline $\begin{array}{l}\text { IVISEM } \\
\text { Total }\end{array}$ & & & & & & & & 1 \\
\hline
\end{tabular}

En la Tabla 2 se muestran las correlaciones entre las puntuaciones observadas de las subescalas del IVISEM, observándose que, si bien las distintas escalas están correlacionadas, en mayor o menor grado, sin embargo, en ningún caso se observan valores por e ncima de .70 , lo que podría comprometer la capacidad discriminativa de las escalas. Por otra parte, la contribución de las distintas subescalas a la puntuación total está compensada siendo las que mayor peso tienen la de cuidados y la de biología y aptitudes, y las que menos la de pareja y neosexismo. 
Tabla 3. Correlaciones entre el IVISEM y el ASI

\begin{tabular}{lcc}
\hline Dimensión & Sexismo Hostil & Sexismo Benevolente \\
\hline Maternidad & $.40^{* *}$ & $.31^{* *}$ \\
Amor romántico y Pareja & $.44^{* *}$ & $.38^{* *}$ \\
Cuidados & $.54^{* *}$ & $.34^{* *}$ \\
Proyección laboral & $.49^{* *}$ & $.34^{* *}$ \\
Actitudes y Sumisión & $.47^{* *}$ & $.28^{* *}$ \\
Biología y Aptitudes & $.53^{* *}$ & $.44^{* *}$ \\
Neosexismo & $.34^{* *}$ & $.77^{* *}$ \\
IVISEM Total & $.65^{* *}$ & $.59^{* *}$ \\
$*_{* *}^{* *} p .01 ; * p<.05$ & &
\end{tabular}

Tabla 4. Correlaciones entre el IVISEM y el CFNI-45-r

\begin{tabular}{lcccccccccc}
\hline Dimensión & Rel & C N & Delg & Fid. & Mod & Rom & Dom & Apar & Dul & CFNI Total \\
\hline $\begin{array}{l}\text { Maternidad } \\
\text { Amor }\end{array}$ & .04 & $.32^{* *}$ & $.14^{* *}$ & $.26^{* *}$ & .03 & $.35^{* *}$ & $.15^{* *}$ & $.07^{* *}$ & $.19^{* *}$ & $.37^{* *}$ \\
$\begin{array}{l}\text { romántico y } \\
\text { Pareja }\end{array}$ & $.06^{*}$ & $.15^{* *}$ & $.14^{* *}$ & $.30^{* *}$ & -.03 & $.55^{* *}$ & .04 & $.07^{* *}$ & $.18^{* *}$ & $.34^{* *}$ \\
$\begin{array}{l}\text { Cuidados } \\
\text { Proyección }\end{array}$ & .03 & $.11^{* *}$ & $.16^{* *}$ & $.28^{* *}$ & $.08^{* *}$ & $.27^{* *}$ & $.11^{* *}$ & .04 & $.16^{* *}$ & $.29^{* *}$ \\
$\begin{array}{l}\text { laboral } \\
\text { Actitudes y }\end{array}$ & .01 & $.18^{* *}$ & $.15^{* *}$ & $.31^{* *}$ & .02 & $.29^{* *}$ & $.10^{* *}$ & .04 & $.14^{* *}$ & $.30^{* *}$ \\
$\begin{array}{l}\text { Sumisión } \\
\text { Biología y }\end{array}$ & .01 & $.05^{*}$ & $.18^{* *}$ & $.26^{* *}$ & $.05^{*}$ & $.23^{* *}$ & .030 & .04 & $.13^{* *}$ & $.23^{* *}$ \\
$\begin{array}{l}\text { Aptitudes } \\
\text { Neosexismo }\end{array}$ & .05 & $.09^{* *}$ & $.18^{* *}$ & $.26^{* *}$ & .04 & $.35^{* *}$ & $.13^{* *}$ & $.10^{* *}$ & $.18^{* *}$ & $.32^{* *}$ \\
IVISEM Total &. .04 & $.12^{* *}$ & $.09^{* * *}$ & $.29^{* *}$ & .04 & $.33^{* *}$ & $.19^{* *}$ & $.12^{* *}$ & $.12^{* *}$ & $.30^{* *}$ \\
\hline
\end{tabular}

**p<.01; *p<.05. En CFNI: Rel=Relaciones; C N=Cuidado de niños; Delg=Preocupación por delgadez; Fid=Fidelidad sexual; Mod=Modestia; Rom=Relaciones románticas; Dom=Doméstica; Apar=Preocupación por Apariencia; Dul=Dulce y Agradable.

Fuentes de evidencia externas: validez de constructo en relación con otras variables

Para analizar la validez de constructo se analiza la relación del IVISEM con dos instrumentos similares (ASI y CFNI-45-r). Como se puede observar en la Tabla 3, las correlaciones entre el ASI y el IVISEM son significativas y prácticamente todas son grandes y muy grandes. La puntuación total del IVISEM muestra una correlación de .65 con la puntuación en Sexismo Hostil y una correlación de .59 con la puntuación en Sexismo Benevolente. Todos los factores del IVISEM muestran elevadas correlaciones con las puntuaciones en ambas formas de sexismo (Hostil y Benevolente), siendo especialmente elevada la correlación entre el factor Neosexismo y el Sexismo Benevolente.

En la Tabla 4 se observa que la puntuación total en IVISEM correlaciona significativamente con la puntuación total en CFNI-45-r con un coeficiente de correlación de Pearson de .43 que representa un tamaño del efecto mediano, observándose, en general, correlaciones estadísticamente significativas entre los componentes de ambos instrumentos.

\section{Discusión}

El IVISEM es un instrumento diseñado para evaluar la socialización diferencial asumida por hombres y mujeres que conlleva una forma de violencia social encubierta que pueden estar sufriendo las mujeres en base a los mandatos de género; es decir, a la interiorización de los roles asignados a hombres y mujeres en función del género asignado al nacer. Se interiorizan los estereotipos basados en el género de tal modo que se convierten en mandatos, en verdades que sin tener una base científica, se dan por válidas precisamente por la intensidad con que se perciben (Barberá \& Martínez, 2004; Dillon, Franco, Kreimer, Struchiner, \& Hannikainen, 2018). Entendemos que, si asumir determinados mandatos de género conlleva consecuencias negativas para quien los asume, esta situación se puede considerar violencia, aunque sea encubierta y que sea la sociedad quien la lleva a cabo y no una persona o grupo concreto (Bourdieu, 1997, 2000; Castellano, Lachica, Molina, \& Villanueva, 2004; Jiménez-Bautista, 2012; Ubillos-Landa, Goiburu-Moreno, Puente- 
Martínez, Pizarro-Ruiz, \& Echeburúa-Odriozola, 2017).

Teniendo en cuenta aquellos mandatos de género que diferentes estudios relacionan con una mayor probabilidad de generar consecuencias negativas para las mujeres se diseñan 7 dimensiones que miden estos mandatos de género (Bareket et al., 2018; Bourdieu, 2000; CarvajalLohr et al., 2016; Casado-Mejía \& GarcíaCarpintero, 2018; Conway, 2013; Delgado et al., 2015; Ferrer-Pérez, \& Bosch-Fiol, 2014, 2019; García-Calvente et al., 2004; Lagarde, 2003; Martínez et al., 2008; Moya et al., 2006; PerezViejo et al., 2011; Simón, 2008; Varela, 2013; Vázquez, 2013).

El instrumento se ha validado en dos grupos de mujeres distintos, lo que ha permitido depurar mejor los ítems para obtener un instrumento final de 35 ítems, fácil y rápido de aplicar y, por tanto, podría ser un instrumento manejable para ser utilizado en diferentes ámbitos socio sanitarios en los que sea conveniente tener en cuenta si la problemática que presentan algunas mujeres podría estar relacionada con esta forma de violencia social encubierta.

Este instrumento ha mostrado excelentes propiedades psicométricas tanto en su fiabilidad por consistencia interna, como en su validez convergente al compararlo con otras medidas similares. Los resultados del análisis del omega jerárquico señalan que el instrumento muestra una fiabilidad adecuada tanto para ser usado de forma multidimensional (cada una de las escalas por separado), como para el uso de la puntuación total. El AFC muestra bondad de ajuste al modelo de siete dimensiones propuesto y el cuestionario cumple con los estándares psicométricos adecuados propuestos por los estándares para test educativos y psicológicos (AERA/APA/NCME, 2014).

Se ha comprobado que el IVISEM muestra una adecuada validez de constructo en relación con otras medidas similares, que miden sexismo ambivalente o conformidad con las normas de género, como son el ASI y el CFNI-45-r. Se han encontrado correlaciones significativas entre todos los factores del IVISEM y los factores de sexismo hostil y benevolente del ASI, que son modos de discriminación que conllevan consecuencias negativas hacia las mujeres (Expósito et al., 1998;
Moya \& Expósito, 2001), por lo que valida el objeto de medida del IVISEM.

Lo mismo ocurre con las correlaciones entre el IVISEM y el CFNI-45-r: se encuentran correlaciones significativas entre el factor Maternidad del IVISEM y el factor Cuidado de niños/as y el de Relaciones Románticas del CFNI45-r; el factor Relaciones de Pareja del IVISEM con el factor Relaciones románticas del CFNI-45r; el factor Cuidado del IVISEM con el factor Cuidado de niños/as y Doméstica del CFNI-45-r; y Actitudes y Sumisión del IVISEM con Fidelidad Sexual del CFNI-45-r. Estos datos, coherentes teóricamente, apoyan, en la línea de diferentes estudios, que existen estereotipos asumidos por las mujeres en cuanto al modo de vivir la maternidad y el cuidado de los niños y niñas y la importancia de las relaciones de pareja (Bernárdez, 2018; Bosch-Fiol et al., 2007; Lagarde, 2003).

Entre las limitaciones del estudio, en primer lugar, habría que tener en cuenta la posible deseabilidad social de las participantes a la hora de responder a este tipo de cuestionarios. En segundo lugar, la recolección de la muestra no fue aleatoria y pudo haber mayor predisposición a responder al inventario de aquellas mujeres más implicadas en la desaparición de las diferencias sociales por razón de género o de aquellas que tengan mayor acceso a redes sociales. Por último, como línea a explorar en futuras investigaciones sería interesante evaluar la capacidad predictiva del IVISEM en relación con la salud de las mujeres que sufren este tipo de violencia social.

El IVISEM viene a cubrir una demanda, tanto académica como social, relacionada con la necesidad de contar con instrumentos de medida fiables y validados que permitan detectar formas de discriminación y violencia hacia las mujeres. Estos instrumentos resultan fundamentales para poder llevar a cabo programas de prevención e intervención (Matud y Aguilera, 2009; PerezViejo et al., 2011; Regal, 2017; Organización Munidal de la Salud, 2018).

\section{Referencias}

American Educational Research Association, American Psychological Association, y National Council on Measurement in 
Education

[AERA/APA/NCME]

(2014). Standards for educational and psychological testing. Washington, DC: American Psychological Association.

Anastasiadou, D., Aparicio, M., Sepúlveda, A. R., \& Sánchez-Beleña, F. (2013). Conformidad con roles femeninos y conductas alimentarias inadecuadas en estudiantes de danza. Revista de Psicopatología y Psicología Clínica, 1(18), 31-43.

Aparicio-García, M. E., \& Alvarado-Izquierdo, J. M. (2019). Is there a "conformity to feminine norms" construct? A bifactor analysis of two short versions of Conformity to Feminine Norms Inventory. Current Psychology, 38, 1110-1120. https://doi.org/10.1007/s12144-018-9815-9

Barberá, E., \& Martínez, I. (coor). (2004). Psicología y género. Madrid: Pearson Educación S.A.

Bareket, O., Kahalon, R., Shnabel, N., \& Glick, P. (2018). The madonna-whore dichotomy: Men who perceive women's nurturance and sexuality as mutually exclusive endorse patriarchy and show lower relationship satisfaction. Sex Roles, 79, 519-532. https://doi.org/10.1007/s11199-018-0895-7

Benson, P. L., \& Vincent, S. (1980). Development and validation of the Sexist Attitudes Toward Women Scale (SATWS). Psychology of Women Quarterly, 5(2), 276-291. https://doi.org/10.1111/j.14716402.1980.tb00962.x

Bernárdez Rodal, A. (2018). Soft power: Heroínas y muñecas en la cultura mediática. Madrid: Fundamentos Ed.

Blum, R. W., Mmari, K., \& Moreau, C. (2017). It begins at 10: How gender expectations shape early adolescence around the world. Journal of Adolescent Health, 61(4), S3-S4. https://doi.org/10.1016/j.jadohealth.2017.07.009

Bonino Méndez, L. (1996). Micromachismos: La violencia invisible en la pareja. Primeras Jornadas de género en la sociedad actual. Valencia: Generalitat Valenciana, 25-45.

Bonino Méndez, L. (2008). Micromachismospoder masculino en la pareja moderna. Voces de hombres por la igualdad. Heterodoxia Boletín de Psicología, No. 100, Noviembre 2010 .
Bosch-Fiol, E., \& Ferrer-Pérez, V. (2013). Nuevo modelo explicativo para la violencia contra las mujeres en la pareja: El modelo piramidal y el proceso de filtraje. Asparkía, 24(Avances y retos actuales para combatir la violencia de genero), 54-67.

Bosch-Fiol, E., Ferrer-Pérez, V. A., García Buades, M. C., Mas Tous, M. C., Navarro Guzmán, C., \& Torrens Espinosa, G. (2007). Del mito del amor romántico a la violencia contra las mujeres en la pareja. In Estudios e investigaciones.Instituto de la Mujer. Ministerio de Igualdad.

Bourdieu, P. (1997). Razones prácticas sobre la teoría de la acción. Barcelona: Anagrama.

Bourdieu, P. (2000). La dominación masculina. Barcelona: Anagrama.

Brown, T. A. (2014). Confirmatory factor analysis for applied research. New York: Guilford Publications.

Carvajal-Lohr, A., Flores-Ramos, M., Marin Montejo, S. I., \& Morales Vidal, C. G. (2016). Los trastornos de ansiedad durante la transición a la menopausia. Perinatología y Reproducción Humana, 30(1), 39-45. https://doi.org/10.1016/j.rprh.2016.03.003

Casado Mejía, R., \& García-Carpintero Muñoz, M. A. (Eds.) (2018). Género y Salud. España: Ed. Díaz de Santos.

Castellano Arroyo, M., Lachica López, E., Molina Rodríguez, A., \& Villanueva de la Torre, $\mathrm{H}$. (2004). Violencia contra la mujer. El perfil del agresor: Criterios de valoración del riesgo. Cuadernos de Medicina Forense, 35, 15-28.

Cohen, J. (1998). Statistical power analysis for the behavioral sciences (2nd ed.). L Erlbaum Associates.

Colás, P. (2007). La construcción de la identidad de género: Enfoques teóricos para fundamentar la investigación e intervención educativa. Revista de Investigación Educativa, 25(1), 151-166.

Conway, J., Bourque, S., \& Scott, J. W. (2013). The concept of gender. In M. Lamas (Ed.), El género: La construcción social de la diferencia sexual (pp. 21-23). Mexico: Purrua.

Corral, Y. (2009). Validez y confiabilidad de los instrumentos de investigación para la recoleccion de datos. Revista Ciencias de La Educación, 19(33), 228-247. 
https://doi.org/10.1093/petrology/egh011

Delegación del Gobierno para la Violencia de Género. (2015). La atención primaria frente a la violencia de género. Necesidades y propuestas. Retrieved from http://www.violenciagenero.igualdad.mpr.gob .es/laDelegacionInforma/pdfs/DGVG_La_Ate ncion_Primaria_VG.PDF

Delgado, A. J., Palma, A. B., \& Rivas, Á. (2015). Neosexism and unfavorable beliefs toward female leader. Revista Electrónica de Investigación y Docencia Creativa, 4, 117122.

Dillon, A. B., Lopes, L., Kreimer, R., Struchiner, N., \& Hannikainen, I. (2018). Gender stereotypes underlie child custody decisions. European Journal of Social Psychology, 49(3), 548-559. https://doi.org/10.1002/ejsp.2523

Etchezahar, E. (2014). La construcción social del género desde la perspectiva de la Teoría de la Identidad Social. Ciencia, Docencia y Tecnología, 25(49), 128-142.

Ellsberg, M., Arango, D. J., Morton, M., Gennari, F., Kiplesund, S., Contreras, M., \& Watts, C. (2015). Prevention of violence against women and girls: What does the evidence say? The Lancet, 385(9977), 1555-1566. https://doi.org/10.1016/S01406736(14)61703-7

Expósito, F., Moya, M. C., \& Glick, P. (1998). Sexismo ambivalente: Medición y correlatos. Revista de Psicología Social, 13(2), 159-169. https://doi.org/10.1174/021347498760350641

Ferrer-Pérez, V. A., \& Bosch-Fiol, E. (2014). Gender violence as a social problem in Spain: Attitudes and acceptability. Sex Roles, 70(1112), 506-521. https://doi.org/10.1007/s11199-013-0322-z

Ferrer-Pérez, V., \& Bosch-Fiol, E. (2019). El género en el análisis de la violencia contra las mujeres en la pareja: De la "ceguera" de género a la investigación específica del mismo. Anuario de Psicología Jurídica, 29(1), 69-76. https://doi.org/10.5093/apj2019a3

Galtung, J. (1990). Cultural violence. Journal of Peace Research, 27(3), 291-305. https://doi.org/10.1177/0022343390027003005 Galtung, J. (2016). La violencia cultural, estructural y directa. Cuadernos de Estrategia, 27(183), 147-168.

García-Calvente, M. D. M., Mateo-Rodríguez, I., \& Maroto-Navarro, G. (2004). El impacto de cuidar en la salud y la calidad de vida de las mujeres. Gaceta sanitaria, 18, 83-92.

García-Moreno, C., Hegarty, K., D’Oliveira, A. F. L., Koziol-McLain, J., Colombini, M., \& Feder, G. (2015a). The health-systems response to violence against women. The Lancet, 385(9977), 1567-1579.

https://doi.org/10.1016/S0140-

6736(14)61837-7

García-Moreno, C., Zimmerman, C., MorrisGehring, A., Heise, L., Amin, A., Abrahams, N., ... Watts, C. (2015b). Addressing violence against women: $\mathrm{A}$ call to action. The Lancet, 385(9978), 16851695. https://doi.org/10.1016/S01406736(14)61830-4

Glick, P., \& Fiske, S. T. (1996). The Ambivalent Sexism Inventory: Differentiating hostile and benevolent sexism. Journal of Personality and Social Psychology, 70(3), 491-512. https://doi.org/10.1037/0022-3514.70.3.491

Grant, J. S., \& Davis, L. L. (1997). Selection and use of content experts for instrument development. Research in Nursing y Health, 20(3), 269-274.

https://doi.org/10.1002/(SICI)1098-

240X(199706)20:3<269::AID-

NUR9>3.0.CO;2-G

Guarderas, P., Larrea, M. D. L., Cuvi, J., Vega, C., Reyes, C., Bichara, T., ... \& Ullaur, K. (2018). Acoso sexual en las universidades ecuatorianas: Validez de contenido de un instrumento de medición. ALTERIDAD. Revista de Educación, 13(2), 214-226. https://doi.org/10.17163/alt.v13n2.2018.05

Hernando, A. (2007). Sexo, género y poder. Breve reflexión sobre algunos conceptos manejados en la Arqueología del Género. Complutum, 18(18), 167-173.

Hu, L. T., \& Bentler, P. M. (1999). Cutoff criteria for fit indexes in covariance structure analysis: Conventional criteria versus new alternatives. Structural equation modeling: A multidisciplinary journal, 6(1), 1-55.

Jiménez-Bautista, F. (2012). Conocer para comprender la violencia: Origen, causas y 
realidad. Convergencia, 19(58), 13-52.

Johnson, M. P. (2006). Conflict and control: Gender symmetry and asymmetry in domestic violence. Violence Against Women, 12(11), 1003-1018.

https://doi.org/10.1177/1077801206293328

Kline, R. B. (2016). Principles and practice of structural equation modeling. New York: The Guilford Press.

Lagarde, M. (1990). Identidad femenina. Secretaría Nacional de Equidad y Género, 25-32.

Lagarde, M. (2003). Los cautiverios de las mujeres. Madresposas, putas, presas y locas. México: Universidad Nacional Autónoma de México.

Lagarde, M. (2006). El derecho humano de las mujeres a una vida libre de violencia. Mujeres, Globalización y Derechos Humanos, 477-534.

Latorre Román, P. Á., López Munera, R., Izquierdo Rus, T., \& García Pinillos, F. (2018). La satisfacción corporal en adultos españoles, influencia del sexo, edad y estado ponderal. Revista Iberoamericana de Diagnóstico y Evaluación - e Avaliação Psicológica, $\quad 47(2), \quad 83-94$. https://doi.org/10.21865/ridep47.2.06

López-Cepero, J., Rodríguez Franco, L., \& Rodríguez Díaz, F. J. (2015). Evaluación de la violencia de pareja. Una revisión de instrumentos de evaluación conductual. Revista Iberoamericana de Diagnóstico y Evaluación - e Avaliação Psicológica, 2(40), 37-50.

Mahalik, J. R., Morray, E. B., Coonerty-Femiano, A., Ludlow, L. H., Slattery, S. M., \& Smiler, A. (2005). Development of the Conformity to Feminine Norms Inventory. Sex Roles, 52(78), 417-435. https://doi.org/10.1007/s11199-005-3709-7

Martínez-Pacheco, A. (2016). La violencia. Conceptualización y elementos para su estudio. Política y Cultura, 46, 7-31.

Martínez, I., Bonilla, A., Gómez, L., \& Bayot, A. (2008). Identidad de género y afectividad en la adolescencia: Asimetrías relacionales y violencia simbólica. Anuario de Psicología, 39(1), 109-118.

Matud, P., \& Aguilera, L. (2009). Roles sexuales y salud mental en una muestra de la población general española. Salud Mental, 32(1), 53-58.

Médicos del Mundo. (2018). Virus Eva. Médicos Del Mundo, (46).

Morillas, D. L., Patró, R. M., \& Aguilar, M. M. (2014). Victimología: Un estudio sobre la víctima y los procesos de victimización (2a). Madrid: Dykinson, S.L.

Moya, M., \& Expósito, F. (2001). Nuevas formas, viejos intereses: Neosexismo en varones españoles. Psicothema, 13(4), 643-649.

Moya, M., Expósito, F., \& Padilla, J. L. (2006). Revisión de las propiedades psicométricas de las versiones larga y reducida de la Escala sobre Ideología de Género. International Journal of Clinical and Health Psychology, 6(3), 709-727.

Muñoz, J. M., \& Echeburúa, E. (2016). Diferentes modalidades de violencia en la relación de pareja: Implicaciones para la evaluación psicológica forense en el contexto legal español. Anuario de Psicologia Jurídica, 26, 2-12.

Novo, M., Herbón, J., \& Amado, B.G. (2016). Género y victimización: Efectos en la evaluación de la violencia psicológica sutil y manifiesta, apego adulto y tácticas de resolución de conflictos. Revista Iberoamericana de $\begin{array}{llll}\text { Psicologia y } \quad \text { Salud, } & \text { 7(2), } & \text { 89-97. }\end{array}$ https://doi.org/10.1016/j.rips.2016.05.002

Organizacion Mundial de la Salud. (2018). Violencia contra la mujer. Retrieved from http://www.who.int/topics/gender_based_viol ence/es/

Perez-Viejo, J. M., Montalvo, A., Escobar, A., Quinteros, A., Sánchez, S., \& Tardón, B. (2011). Violencia de género. Prevención,detección y atención (J. PérezViejo y A. Montalvo, Eds.). Madrid: Ed. Grupo 5.

Regal, R. J. (2017). Características epidemiológicas de los pacientes evaluados por fibromialgia en la Unidad Médica de Valoración de Incapacidades de Madrid. SEMERGEN - Medicina de Familia, 43(1), 28-33.

https://doi.org/https://doi.org/10.1016/j.semer g.2015.12.015

Rodríguez-Blanes, G. M., Vives-Cases, C., Miralles-Bueno, J. J., San Sebastian, M., \& 
Goicolea, I. (2017). Detección de violencia del compañero íntimo en atención primaria de salud y sus factores asociados. Gaceta Sanitaria, 31(5).

https://doi.org/10.1016/j.gaceta.2016.11.008

Sackett, L. A., \& Sounders, D. G. (2001). The impact of different forms of psychological abuse in battered women. In O'Leary y Mairuo (Ed.), Psychological abuse in violent domestic relations (pp. 197-210). New York: Springer.

Segato, R. L. (2003). Las estructuras elementales de la violencia. In Curso de verano sobre violencia de género. Madrid: Universidad Complutense de Madrid.

Simón, M. E. (2008). Hijas de la igualdad, heredeas de injusticias. Madrid: Narcea S.A.

Temmerman, M. (2015). Research priorities to address violence against women and girls. The Lancet, 385(9978), e38-e40. https://doi.org/https://doi.org/10.1016/S01406736(14)61840-7

Ubillos-Landa, S., Goiburu-Moreno, E., PuenteMartínez, A., Pizarro-Ruiz, J.-P., \& Echeburúa-Odriozola, E. (2017). Assessment of distorted thoughts about women and violence of basque-speaking secondary school students. Revista de Psicodidáctica (English Ed.), 22(1), 1-8. https://doi.org/10.1387/RevPsicodidact.16124

Varela, N. (2013). Feminismo para principiantes. Barcelona: Edicioens B.S.A.

Vázquez, A. (2013). Depresión. Diferencias de género. 17(3), 1-21.

Vinagre González, A. M. (2019). Violencia social encubierta hacia la mujer y su repercusión en la salud (Tesis Doctoral, Universidad Complutense de Madrid). 


\section{Anexo I.}

\section{VIOLENCIA SOCIAL ENCUBIERTA HACIA LA MUJER (IVISEM)}

Este Inventario tiene como objetivo conocer cómo es su vivencia o experiencia en aspectos relacionados con el hecho de ser mujer y el papel que usted como mujer ocupa en la sociedad. Por favor, indique en qué modo cada una de las siguientes afirmaciones refleja su experiencia en este sentido, utilizando la siguiente escala: 1 Totalmente en desacuerdo, 2 Bastante en desacuerdo, 3 Ni de acuerdo ni en desacuerdo, 4 Bastante de acuerdo y 5 Totalmente de Acuerdo. No existen respuestas correctas ni incorrectas. Tenga en cuenta que lo importante es conocer cómo es su vivencia o experiencia, no su opinión sobre cómo deberían ser las cosas. Por favor responda con sinceridad. El cuestionario es anónimo.

\begin{tabular}{|c|c|c|c|c|c|c|}
\hline & & 1 & 2 & 3 & 4 & 5 \\
\hline 1 & Las madres tienen un vínculo especial con los/as hijos/as que los padres no tienen. & & & & & \\
\hline 2 & Lo ideal es encontrar una pareja con quien ser feliz para siempre. & & & & & \\
\hline 3 & $\begin{array}{l}\text { Cuando hay que llevar a los/as niños/as al médico, las madres entienden y llevan a cabo las } \\
\text { indicaciones mejor que los padres. }\end{array}$ & & & & & \\
\hline 4 & $\begin{array}{l}\text { Si hay que elegir entre la mujer y el hombre para cuidar de los/as hijos/as, es más conveniente que } \\
\text { sea ella la que renuncie a parte de su vida profesional. }\end{array}$ & & & & & \\
\hline 5 & Los hombres son los que suelen tomar las decisiones económicas importantes. & & & & & \\
\hline 6 & En general las mujeres tienen peor capacidad espacial. Por ejemplo, manejan peor un mapa. & & & & & \\
\hline 7 & $\begin{array}{l}\text { En realidad, las feministas sólo buscan la igualdad, no la superioridad de la mujer con respecto al } \\
\text { hombre. }\end{array}$ & & & & & \\
\hline 8 & $\begin{array}{l}\text { Si hay que elegir, es la madre quien debería darse de baja por maternidad los primeros meses. El/la } \\
\text { bebé la necesita más que al padre. }\end{array}$ & & & & & \\
\hline 9 & No creo que sea importante tener pareja para ser feliz. & & & & & \\
\hline 10 & $\begin{array}{l}\text { Una mujer cuida mejor de los/as hijos/as y de los/las mayores porque tiene mayor capacidad de } \\
\text { renuncia y sacrificio. }\end{array}$ & & & & & \\
\hline 11 & La ambición profesional debería estar supeditada a su familia en el caso de una mujer. & & & & & \\
\hline 12 & Cuidar el aspecto físico es más propio de mujeres que de hombres. & & & & & \\
\hline 13 & Las mujeres tienen más problemas de ansiedad por sus cambios hormonales. & & & & & \\
\hline 14 & $\begin{array}{l}\text { Algunas mujeres están ocupando cargos para los que no están preparadas por la moda de la } \\
\text { paridad o igualdad. }\end{array}$ & & & & & \\
\hline 15 & Ser madre es probablemente el acontecimiento más importante en la vida de una mujer. & & & & & \\
\hline 16 & Una forma de demostrar amor hacia mi pareja es ser capaz de renunciar a cosas por él. & & & & & \\
\hline 17 & En general el hombre es más para sí mismo, la mujer es más para los demás. & & & & & \\
\hline 18 & $\begin{array}{l}\text { Un cargo que implique pasar muchas horas fuera de casa no será adecuado para una mujer si tiene } \\
\text { hijos/as pequeños/as. }\end{array}$ & & & & & \\
\hline 19 & La mujer normalmente es más sumisa que el hombre. & & & & & \\
\hline 20 & $\begin{array}{l}\text { Es normal que haya más mujeres psicólogas que hombres, ya que la mujer suele tener mayor } \\
\text { capacidad de empatía. }\end{array}$ & & & & & \\
\hline 21 & Se está exagerando en cuanto a la desigualdad entre hombres y mujeres. & & & & & \\
\hline 22 & La lactancia materna es la mejor forma de unión con el bebé. & & & & & \\
\hline 23 & En una relación de pareja la renuncia y el sacrificio no son importantes. & & & & & \\
\hline 24 & $\begin{array}{l}\text { Las hijas suelen estar mejor preparadas que los hijos para atender a su padre o madre si estos son } \\
\text { dependientes. }\end{array}$ & & & & & \\
\hline 25 & $\begin{array}{l}\text { Es difícil que una mujer acepte puestos de responsabilidad en una empresa si ello implica pasar } \\
\text { muchas horas fuera de casa y tiene hijos/as pequeños/as. }\end{array}$ & & & & & \\
\hline 26 & $\begin{array}{l}\text { Es normal que una chica se haga respetar más que un chico y se muestre más prudente en } \\
\text { conductas de tipo sexual. }\end{array}$ & & & & & \\
\hline 27 & La mujer es menos fuerte físicamente que el hombre. & & & & & \\
\hline 28 & Actualmente los hombres están siendo acosados por las feministas. & & & & & \\
\hline 29 & $\begin{array}{l}\text { Una mujer debería anteponer la lactancia materna y sus beneficios a posibles. inconvenientes que } \\
\text { esto pueda generarle como dolor, incomodidad, etc. }\end{array}$ & & & & & \\
\hline 30 & Es importante encontrar a "tu media naranja”, aunque eso implique renunciar a algunas cosas. & & & & & \\
\hline 31 & $\begin{array}{l}\text { Cuando los padres son mayores, es mejor que vivan cerca de una hija, para que esta pueda estar } \\
\text { pendiente de ellos. }\end{array}$ & & & & & \\
\hline 32 & $\begin{array}{l}\text { Si hay que pedir reducción de jornada por atender a una persona mayor en casa, es más } \\
\text { conveniente que lo haga la mujer. }\end{array}$ & & & & & \\
\hline 33 & Es más importante mostrarse prudente en una mujer que en hombre. & & & & & \\
\hline 34 & La mujer es más sensible que el hombre. & & & & & \\
\hline 35 & Hoy día el feminismo ya no es necesario. & & & & & \\
\hline
\end{tabular}

